Network Working Group

Request for Comments: 3251

Category: Informational
B. Rajagopalan

Tellium, Inc.

1 April 2002

Electricity over IP

Status of this Memo

This memo provides information for the Internet community. It does not specify an Internet standard of any kind. Distribution of this memo is unlimited.

Copyright Notice

Copyright (C) The Internet Society (2002). All Rights Reserved.

Abstract

Mostly Pointless Lamp Switching (MPLamps) is an architecture for carrying electricity over IP (with an MPLS control plane). According to our marketing department, MPLamps has the potential to dramatically lower the price, ease the distribution and usage, and improve the manageability of delivering electricity. This document is motivated by such work as SONET/SDH over IP/MPLS (with apologies to the authors). Readers of the previous work have been observed scratching their heads and muttering, "What next?". This document answers that question.

This document has also been written as a public service. The "SubIP" area has been formed to give equal opportunity to those working on technologies outside of traditional IP networking to write complicated IETF documents. There are possibly many who are wondering how to exploit this opportunity and attain high visibility. Towards this goal, we see the topics of "foo-over-MPLS" (or MPLS control for random technologies) as highly amenable for producing a countless number of unimplementable documents. This document illustrates the key ingredients that go into producing any "fooover-MPLS" document and may be used as a template for all such work.

1. Conventions used in this document

The key words "MUST", "MUST NOT", "DO", "DON'T", "REQUIRED", "SHALL", "SHALL NOT", "SHOULD", "SHOULD NOT", "RECOMMENDED", "MAY", "MAY BE" and "OPTIONAL" in this document do not mean anything. 
2. Pre-requisite for reading this document

While reading this document, at various points the readers may have the urge to ask questions like, "does this make sense?", "is this feasible?," and "is the author sane?". The readers must have the ability to suppress such questions and read on. Other than this, no specific technical background is required to read this document. In certain cases (present document included), it may be REQUIRED that readers have no specific technical background.

3. Introduction

It was recently brought to our attention that the distribution network for electricity is not an IP network! After absorbing the shock that was delivered by this news, the following thoughts occurred to us:

1. Electricity distribution must be based on some outdated technology (called "Legacy Distribution system" or LDS in the rest of the document).

2. An LDS not based on the Internet technology means that two different networks (electricity and IP) must be administered and managed. This leads to inefficiencies, higher cost and bureaucratic foul-ups (which possibly lead to blackouts in California. We are in the process of verifying this using simulations as part of a student's MS thesis).

3. The above means that a single network technology (i.e., IP) must be used to carry both electricity and Internet traffic.

4. An internet draft must be written to start work in this area, before someone else does.

5. Such a draft can be used to generate further drafts, ensuring that we (and CCAMP, MPLS or another responsible working group) will be busy for another year.

6. The draft can also be posted in the "white papers" section of our company web page, proclaiming us as revolutionary pioneers.

Hence the present document.

4. Terminology

MPLampS: Mostly Pointless Lamp Switching - the architecture introduced in this document.

Lamp: An end-system in the MPLamps architecture (clashes with the IETF notion of end-system but of course, we DON'T care).

LER: Low-voltage Electricity Receptor - fancy name for "Lamp". 


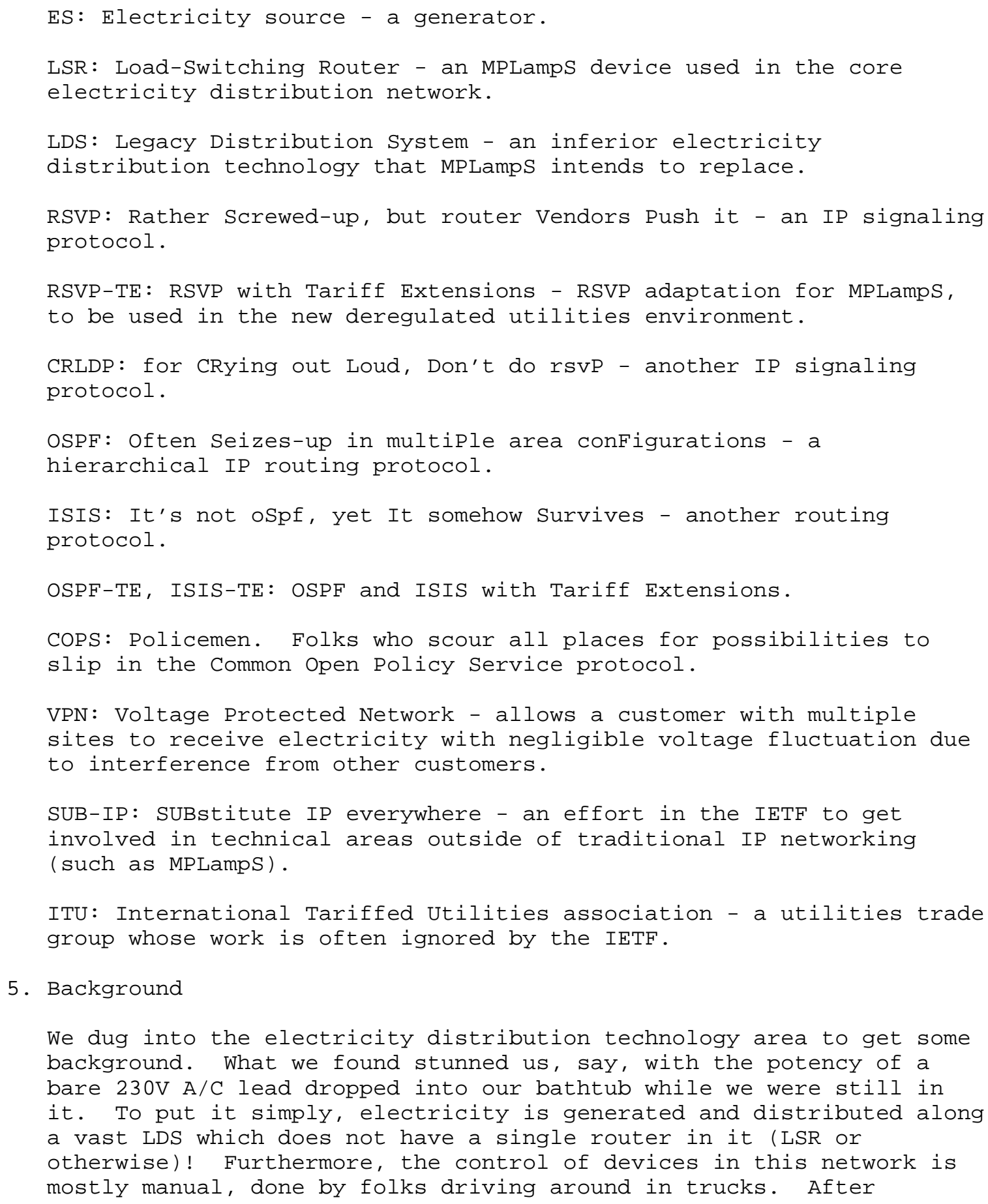


wondering momentarily about how such a network can exist in the 21st century, we took a pencil and paper and sketched out a scenario for integrating the LDS network with the proven Internet technology. The fundamental points we came up with are:

1. IP packets carry electricity in discrete, digitized form.

2. Each packet would deliver electricity to its destination (e.g., a device with an IP address) on-demand.

3. MPLS control will be used to switch packets within the core LDS, and in the edge premises. The architecture for this is referred to as Mostly-Pointless Lamp Switching (MPLamps).

4. The MPLamps architectural model will accommodate both the overlay model, where the electricity consuming devices (referred to as "lamps") are operated over a distinct control plane, and the peer model, in which the lamps and the distribution network use a single control plane.

5. RSVP-TE (RSVP with Tariff Extensions) will be used for establishing paths for electricity flow in a de-regulated environment.

6. COPS will be used to support accounting and policy.

After jotting these points down, we felt better. We then noted the following immediate advantages of the proposed scheme:

1. Switches and transformers in the LDS can be replaced by LSRS, thereby opening up a new market for routers.

2. Electricity can be routed over the Internet to reach remote places which presently do not have electricity connections but have only Internet kiosks (e.g., rural India).

3. Electrical technicians can be replaced by highly paid IP network administrators, and

4. The IETF can get involved in another unrelated technology area.

In the following, we describe the technical issues in a vague manner.

6. Electricity Encoding

The Discrete Voltage Encoding (DVE) scheme has been specified in ITU standard G.110/230V [2] to digitize electrical voltages. In essence, an Electricity source (ES) such as a generator is connected to a DV encoder that encodes the voltage and current, and produces a bit stream. This bit stream can be carried in IP packets to various destinations (referred to as LERs - Low-voltage Electricity Receptors) on-demand. At the destination, a DV decoder produces the right voltage and current based on the received bit stream. It is to be determined whether the Real-time Transport Protocol (RTP) can be 
used for achieving synchronization and end-to-end control. We leave draft writing opportunities in the RTP area to our friends and colleagues.

\section{MPLamps Architecture}

\subsection{Overview}

In an LDS, the long-haul transmission of electricity is at high voltages. The voltage is stepped down progressively as electricity flows into local distribution networks and is finally delivered to LERs at a standard voltage (e.g., 110V). Thus, the LDS is a hierarchical network. This immediately opens up the possibility of OSPF and ISIS extensions for routing electricity in a transmission network, but we'll contain the urge to delve into these productive internet draft areas until later. For the present, we limit our discussion merely to controlling the flow of electricity in an IPbased distribution network using MPLamps.

Under MPLamps, a voltage is equated to a label. In the distribution network, each switching element and transformer is viewed as a loadswitching router (LSR). Each IP packet carrying an electricity flow is assigned a label corresponding to the voltage. Electricity distribution can then be trivially reduced to the task of label (voltage) switching as electricity flows through the distribution network. The configuration of switching elements in the distribution network is done through RSVP-TE to provide electricity on demand.

We admit that the above description is vague and sounds crazy. The example below tries to add more (useless) details, without removing any doubts the reader might have about the feasibility of this proposal:

Example: Turning on a Lamp

It is assumed that the lamp is controlled by an intelligent device (e.g, a (light) switch with an MPLamps control plane). Turning the lamp on causes the switch to issue an RSVP-TE request (a PATH message with new objects) for the electricity flow. This PATH message traverses across the network to the ES. The RESV message issued in return sets up the label mappings in LSRs. Finally, electricity starts flowing along the path established. It is expected that the entire process will be completed within a few seconds, thereby giving the MPLamps architecture a distinct advantage over lighting a candle with a damp match stick. 


\subsection{Overlay vs Peer Models}

As noted before, there are two control plane models to be considered. Under the overlay model, the lamps and the distribution network utilize distinct control planes. Under the peer model, a single control plane is used. A number of arguments can be made for one model versus the other, and these will be covered in the upcoming framework document. We merely observe here that it is the lamp vendors who prefer the peer model against the better judgement of the LSR vendors. We, however, want to please both camps regardless of the usefulness of either model. We therefore note here that MPLampS supports both models and also migration scenarios from overlay to peer.

\subsection{Routing in the Core Network}

The above description of the hierarchical distribution system immediately opens up the possibility of applying OSPF and ISIS with suitable extensions. The readers may rest assured that we are already working on such concepts as voltage bundling, multi-area tariff extensions, insulated LSAs, etc. Future documents will describe the details.

\subsection{Voltage Protected Networks (VPNs)}

VPNs allow a customer with multiple sites to get guaranteed electricity supply with negligible voltage fluctuations due to interference from other customers. Indeed, some may argue that the entire MPLamps architecture may be trashed if not for the possibility of doing VPNs. Whatever be the case, VPNs are a hot topic today and the readers are forewarned that we have every intention of writing several documents on this. Specifically, BGP-support for VPNs is an area we're presently eyeing with interest.

8. Multicast

It has been observed that there is a strong spatial and temporal locality in electricity demand. ITU Study Group 55 has studied this phenomenon for over a decade and has issued a preliminary report. This report states that when a lamp is turned on in one house, it is usually the case that lamps are turned on in neighboring houses at around the same time (usually at dusk) [3]. This observation has a serious implication on the scalability of the signaling mechanism. Specifically, the distribution network must be able to handle tens of thousands of requests all at once. The signaling load can be reduced if multicast delivery is used. Briefly, a request for electricity is not sent from the lamp all the way to an ES, but is handled by the first LSR that is already in the path to another lamp. 
Support for this requires the application of multicast routing protocols together with RSVP-TE shared reservation styles and the development of MPLamps multicast forwarding mode. We are currently studying the following multicast routing protocol:

o DVMRP: Discrete Voltage Multicast Routing Protocol - this protocol works over existing voltage routing protocols but the danger here is that electricity is delivered to all lamps when any one lamp is turned on. Indeed, the switching semantics gets annoying - all lamps get turned on periodically and those not needed must be switched off each time manually.

Other protocols we will eventually consider are Current-Based Tree (CBT) and Practically Irrelevant Multicast (PIM). An issue we are greatly interested in is multicast scope: we would like support for distributing electricity with varying scope, from lamps within a single Christmas tree to those in entire cities. Needless to say, we will write many detailed documents on these topics as time progresses.

9. Security Considerations

This document MUST be secured in a locked cabinet to prevent it from being disposed off with the trash.

10. Summary

This document described the motivation and high level concepts behind Mostly Pointless Lamp Switching (MPLampS), an architecture for electricity distribution over IP. MPLamps utilizes DVE (discrete voltage encoding), and an MPLS control plane in the distribution network. Since the aim of this document is to be a high-visibility place-holder, we did not get into many details of MPLamps. Numerous future documents, unfortunately, will attempt to provide these details.

11. References

1. A. Malis, et al., "SONET/SDH Circuit Emulation Service Over MPLS (CEM) Encapsulation", Internet Draft, Work in Progress.

2. International Tarriffed Utilities association draft standard, ITU G.110/230V, "Discrete Voltage Encoding", March, 1999.

3. International Tarriffed Utilities association technical report, ITU (SG-55) TR-432-2000, "Empirical Models for Energy Utilization", September, 2000 . 
12. Disclaimer

The opinions expressed in this document are solely the author's.

Company's opinions, as always, are proprietary and confidential and may be obtained under appropriate NDAs.

13. Author's Address

Bala Rajagopalan

Tellium, Inc.

2 Crescent Place

Ocean Port, NJ 07757

Phone: 732-923-4237

EMail: brajadtellium.com 
14. Full Copyright statement

Copyright (C) The Internet Society (2002). All Rights Reserved.

This document and translations of it may be copied and furnished to others, and derivative works that comment on or otherwise explain it or assist in its implementation may be prepared, copied, published and distributed, in whole or in part, without restriction of any kind, provided that the above copyright notice and this paragraph are included on all such copies and derivative works. However, this document itself may not be modified in any way, such as by removing the copyright notice or references to the Internet society or other Internet organizations, except as needed for the purpose of developing Internet standards in which case the procedures for copyrights defined in the Internet Standards process must be followed, or as required to translate it into languages other than English.

The limited permissions granted above are perpetual and will not be revoked by the Internet society or its successors or assigns.

This document and the information contained herein is provided on an "AS IS" basis and THE INTERNET SOCIETY AND THE INTERNET ENGINEERING TASK FORCE DISCLAIMS ALL WARRANTIES, EXPRESS OR IMPLIED, INCLUDING BUT NOT LIMITED TO ANY WARRANTY THAT THE USE OF THE INFORMATION HEREIN WILL NOT INFRINGE ANY RIGHTS OR ANY IMPLIED WARRANTIES OF MERCHANTABILITY OR FITNESS FOR A PARTICULAR PURPOSE.

Acknowledgement

Funding for the RFC Editor function is currently provided by the Internet society. 